\title{
AVALIAÇÃO DA INCRUSTAÇÃO NA BOMBA DE FUNDO EM POÇOS PRODUTORES DE PETRÓLEO E GÁS: ESTUDO DE CASO
}

\author{
J. D. Cunha ${ }^{1 *}$, F. N. Silva ${ }^{2}$, A. F. F. Barbosa ${ }^{3}$ e D. R. Silva ${ }^{1}$ \\ ${ }^{1}$ Universidade Federal Rural do Semi-Árido - UFERSA \\ ${ }^{2}$ Universidade Federal do Rio Grande do Norte - UFRN \\ ${ }^{3}$ Universidade Federal do Maranhão - UFMA \\ jardel.dantas@ufersa.edu.br*
}

Artigo submetido em março/2015 e aceito em setembro/2016

DOI: $10.15628 /$ holos.2016.2878

\section{RESUMO}

$\mathrm{Na}$ indústria petrolífera a problemática da formação de incrustação provoca uma série de danos, entre os quais podemos citar a redução na produção de líquidos e consequentemente de petróleo, havendo também a elevação dos custos para a produção deste. O estudo das incrustações é então de suma importância para o conhecimento do mecanismo de sua formação e para a escolha do método de prevenção e/ou remoção. Técnicas de extração Soxhlet, EFRX, DRX e MEV, foram utilizadas para caracterização das amostras. Através da análise dos resultados foi possível identificar: incrustação tipo ferruginosa, cuja sua formação está associada à presença de agentes corrosivos; e tipo carbonática, sendo sua formação influenciada pela concentração de íons de cálcio e bicarbonato, dissolvidos na água, uma vez que tal formação é comum em poços que apresentam teores elevados desses íons, que também é favorecida pela diminuição da pressão ou aumento da temperatura no reservatório.

PALAVRAS-CHAVE: Incrustação, Elevação Artificial, Corrosão, Caracterização.

\section{SCALE ASSESSMENT IN DOWNHOLE PUMP OF THE WELLS PRODUCING OIL AND GAS: CASE STUDY}

\begin{abstract}
In the oil industry the problem of scale formation causes a lot of damage, among which we can mention the reduction in production of liquid and consequently oil production, there is also the increased costs to the oil production. The study of the scale is extremely important to the understanding of the formation mechanism and the choice of prevention and removal method of the scales. Techniques such as, Soxhlet extraction, XRF, SEM and XRD were used to characterize the samples.
\end{abstract}

Analyzing the results were identified: ferruginous scale, which its formation is associated with the presence of corrosive agents; and carbonate scale, your formation is influenced by the concentration of calcium ions and bicarbonate ions dissolved in the water, this type of formation is common in wells that have a high concentration of these ions, which is also favored by relief pressure and increased temperature in the reservoir.

KEYWORDS: Scale, Articial lift, Corrosion, Characterization. 


\section{INTRODUÇÃO}

Os sistemas de elevação artificial são necessários na vida de um campo produtor, quando as pressões de reservatório diminuem e a produtividade dos poços entra em declínio dado que os fluidos não conseguem energia suficiente para vencer, a coluna hidrostática do fluido na coluna de produção, as perdas por fricção, as perdas nas restrições, as perdas na linha de produção e a pressão nos equipamentos de separação.

Segundo TAKÁCKS (2002), considerando os poços que produzem com elevação artificial o bombeio mecânico compreende cerca de $80 \%$ dos poços e os demais são atribuídos às técnicas de elevação de gás-lift, bombeio de cavidade progressiva (BCP) e bombeio elétrico submerso (BCS).

A seleção do método de elevação artificial esta relacionado ao tipo de completação, diâmetro do revestimento, se o mesmo é direcional, a existência de energia disponível, a profundidade do poço, ao índice de produtividade do poço, produção de areia e parafina, ao grau ${ }^{\circ} \mathrm{API}$ do óleo e o potencial de incrustação da água CLEGG et al. (1992), HEIN (1996) e JAYASEKERA (2000).

O bombeio mecânico com hastes (BM) é o método de elevação artificial mais empregado no mundo, podendo o mesmo ser empregado para elevar vazões médias de poços rasos ou baixas vazões para grandes profundidades (THOMAS, 2004).

Durante a produção de petróleo, é comum o surgimento de processos de natureza corrosiva, depósitos inorgânicos e orgânicos, hidratos e formação de emulsão estável, podendo haver comprometimento no escoamento do petróleo e até mesmo, obstrução das colunas, tubulações e afetar o funcionamento dos equipamentos de superfície e subsuperfície. As incrustações comuns na indústria do petróleo podem ser encontradas: rocha reservatório BOIM (2003); canhoneados e equipamentos de subsuperfície (WOOD, 2006) tais como: gravel packing, coluna de produção, dentre outros.

À medida que os campos produtores vão sendo depletados, técnicas de estimulação como a injeção de água vão sendo implantadas com o objetivo de manter a pressão no interior do reservatório assim como também deslocar o óleo na direção dos poços produtores. Segundo DAHER (2003) a formação de incrustações ocorre em função da incompatibilidade química entre a água de injeção rica em íons sulfatos $\left(\mathrm{SO}_{4}^{-2}\right)$, cloretos $\left(\mathrm{Cl}^{-}\right)$, bicarbonato $\left(\mathrm{HCO}_{3}^{-}\right)$e a água de formação rica em cátions como magnésio $\left(\mathrm{Mg}^{+2}\right)$, cálcio $\left(\mathrm{Ca}^{+2}\right)$, sódio $\left(\mathrm{Na}^{+2}\right)$, bário $\left(\mathrm{Ba}^{+2}\right)$ e estrôncio $\left(\mathrm{Sr}^{+2}\right)$. Estas águas quando submetidas a condições termodinâmicas adequadas, faz com que sejam depositados compostos insolúveis tais como $\mathrm{BaSO}_{4}$ (Equação 1) (sulfato de bário ou Barita), $\mathrm{SrSO}_{4}$ (Equação 2) (sulfato de estrôncio ou Celestina), $\mathrm{CaSO}_{4}$ (Equação 3) (sulfato de cálcio ou Anidrita) e $\mathrm{CaCO}_{3}$ (Equação 4) (Carbonato de cálcio ou Calcita) MARQUES et al. (2001). No caso da formação da calcita a mesma apresenta um mecanismo diferente que envolve um desequilíbrio termodinâmico entre o dióxido de carbono $\left(\mathrm{CO}_{2}\right)$ e o bicarbonato $\left(\mathrm{HCO}_{3}^{-}\right)$. Dentre estes compostos, o sulfato de bário é o mais problemático por ser o de menor solubilidade e o de mais difícil remoção (BOIM, 2003).

$$
\mathrm{Ba}^{+2}+\mathrm{SO}_{4}^{2} \rightarrow \mathrm{BaSO}_{4} \downarrow
$$




$$
\begin{aligned}
& \mathrm{Sr}^{+2}+\mathrm{SO}_{4}{ }^{2} \rightarrow \mathrm{SrSO}_{4} \downarrow \\
& \mathrm{Ca}^{+2}+\mathrm{SO}_{4}{ }^{2} \rightarrow \mathrm{CaSO}_{4} \downarrow \\
& \mathrm{Ca}^{+2}+2 \mathrm{HCO}_{3} \rightarrow \mathrm{CaCO}_{3} \downarrow+\mathrm{CO}_{2} \uparrow+\mathrm{H}_{2} \mathrm{O}
\end{aligned}
$$

Assim, no poço produtor, a pressão e temperatura diminuem ao longo da coluna até a superfície, mudando as condições termodinâmicas de precipitação nos componentes da coluna (RAMTAD, 2005). Desta forma as incrustações (ou scale) podem ser definidas como compostos químicos de natureza inorgânica que se encontram inicialmente solúveis em soluções salinas, as quais, quando submetidas a mudanças de pressão, temperatura e concentração mudam suas condições de solubilidade (DEMADIS, 2007).

Para avaliar os produtos de incrustações formados e mostrar a importância das técnicas de caracterização o presente trabalho tem como objetivo analisar a composição das incrustações coletadas e sua morfologia.

\section{METODOLOGIA}

\subsection{Coleta das amostras}

As amostras foram coletadas nas válvulas de passeio durante as operações de intervenção na coluna de produção de dois poços que são estimulados pela técnica de injeção de água. As amostras foram denominadas de AM1 e AM2.

\subsection{Extração Soxhlet}

A extração Soxhlet foi realizada no equipamento da marca Avanti modelo 2055-Soxtec, com copos e sistemas limpos e secos. Foi extraída $5 \mathrm{~g}$ de amostra, sendo representativa quando comparado a amostra total. O solvente utilizado no procedimento de extração foi clorofórmio, onde o mesmo é mais adequado por apresentar menor custo e baixa toxicidade. Foi utilizado 60 $\mathrm{ml}$ de clorofórmio, em que o ciclo de extração foi realizado a temperatura de $140^{\circ} \mathrm{C}$ durante 120 minutos. Após extração, a amostra foi pulverizada em moinho planetário RETSCH PM 200 com 500 rpm, durante 10 minutos, até amostra obter granulometria próxima de 100 \# (mesh) (Moura, 2006).

\subsection{Espectrometria por fluorescência de raios X (EFRX)}

A análise de EFRX foi realizada num equipamento da marca Shimadzu modelo XRF-1800 no modo semi-quantitativo no qual é empregada a metodologia de dispersão por comprimento de onda (WD-XRF). O equipamento possui limite de deteç̧ão do sódio ( $\mathrm{Na} \rightarrow \mathrm{Z}=11$ ) ao urânio ( $U \rightarrow Z=92$ ) utilizando radiação $\operatorname{RhK} \alpha(\lambda=0,615 \AA \hat{)})$. Na preparação da amostra foi utilizada a prensagem da amostra com ácido bórico, utilizando uma proporção de 6:1 (ácido bórico:amostra) com dois ciclos de prensagem de 60 segundo com uma carga de $30 \mathrm{kPa}$ 


\subsection{Difração de raios X (DRX)}

A difração de raios $X$ foi realizada em um equipamento da marca Shimadzu modelo LabX XRD - 6000, com radiação CuKa ( $\lambda=1,5418$ Á). O difratograma obtido foi analisado pelos programas Search Match v.3.01 e Basic Process da Shimadzu com banco de dados do JCPDS (Joint Committee on Powder Diffraction Standards). Dessa forma, dependendo das fases cristalinas presentes têm-se o conhecimento dos produtos de incrustação e/ou corrosão.

\subsection{Microscopia eletrônica de varredura (MEV)}

A análise por MEV foi realizada num equipamento da marca Philips modelo XL-30 ESEM em modo baixo vácuo (modo ambiental), nesse modo não houve necessidade de se metalizar a amostra.

Fragmentos de cada amostra foram extraídos (Soxhlet), removendo toda parte orgânica, para não haver contaminação do equipamento. Nesta análise, as amostras não foram pulverizadas, possibilitando o estudo da formação da incrustação, da morfologia presente nela e da composição química através do EDS (Energy Dispersive Spectrometry).

\section{RESULTADOS E DISCUSSÕES}

\subsection{Extração Soxhlet}

De acordo com a Tabela 2 são apresentados os resultados obtidos na extração Soxhlet para as amostras AM1 e AM2. As frações orgânicas foram de 5,56 \% e 1,26 \%, respectivamente, o que indica pouca ou nenhuma presença de hidrocarbonetos nas amostras em estudo. Salientando que, para cada amostra, foram pesados em torno de $5 \mathrm{~g}$ e que tais teores são calculados com base nestes valores.

Tabela 2 - Resultados dos teores inorgânico ( $\left.T_{\text {inorg}}\right)$ e orgânico ( $T_{\text {org }}$ ) após extração Soxhlet.

\begin{tabular}{c|c|c}
\hline Amostra & $\mathrm{T}_{\text {inorg }}(\%)$ & $\mathrm{T}_{\text {org }}(\%)$ \\
\hline AM1 & 94,37 & 5,56 \\
\hline AM2 & 98,09 & 1,26 \\
\hline
\end{tabular}

Observa-se, na Figura 1a, que o resíduo coletado apresenta cor bege com tom escuro, indicando que a existência de hidrocarbonetos conforme apresenta os dados de extração. A amostra já extraída e pulverizada (Figura1b) apresentou cor aparente diferente da observada antes do processo de extração.
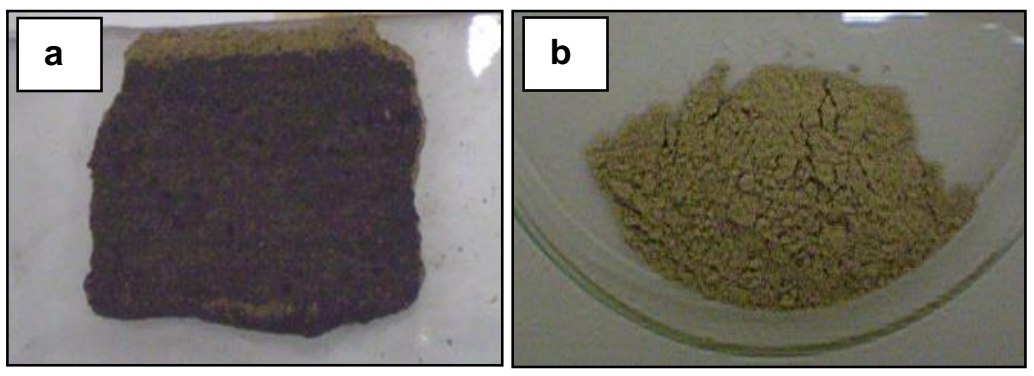

Figura 1: Amostras AM1 e AM2 - a) aspecto da amostra AM1 antes da extração Soxhlet e b) amostra já extraída e pulverizada. 


\subsection{EFRX}

O resultado de fluorescência (Tabela 3 ) apresentou como elementos majoritários: cálcio (Ca-88,02 \%) e ferro (Fe-5,71\% \%) para a amostra AM1 e os demais elementos apresentaram concentração abaixo de $1 \%$. Através deste resultado também é possível inferir que a amostra AM1 se trata de um produto de incrustação formado na bomba de fundo.

Tabela 3 - Resultado de EFRX (modo semi-quantitativo) referente às amostras AM1 e AM2.

A amostra AM2, o resultado de EFRX (Tabela 3) mostrou o cálcio (Ca-95,34 \%) e manganês (Mn-1,26\%) como elementos majoritários e os demais elementos apresentaram concentração abaixo de $1 \%$, sendo estes classificados como traços. Deste modo é observado que a amostra AM2 trata-se também de um produto de incrustação, que se formou na bomba de fundo. Em ambos os casos, AM1 e AM2 apresentaram como a cor bege decorrente a presença do cálcio.

\subsection{DRX}

Através da análise do difratograma (Figura 3) foram detectadas duas fases cristalinas, as quais apresentaram segundo banco de dados JCPDS (Tabela 4) as fases: Carbonato de cálcio $\left(\mathrm{CaCO}_{3}\right)$ e Hidróxido de ferro $(\mathrm{FeOOH})$. As fases presentes confirmam os altos teores de cálcio e ferro que foram detectadas pelas técnicas de EFRX (Tabela 3)

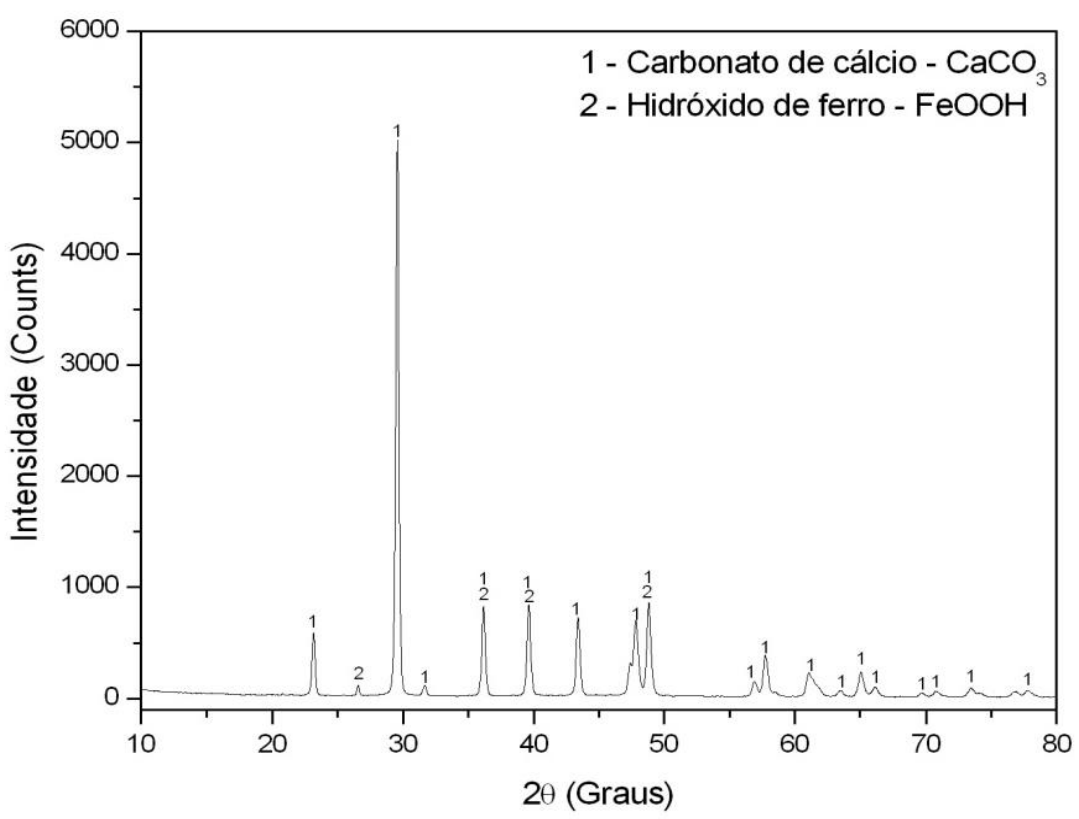

Figura 3 - Difratograma das principais fases presentes na amostra AM1.

No difratograma da amostra AM1 (Figura 3) foi detectada uma fase cristalina, a qual apresentou segundo o banco de dados JCPDS (Tabela 4) a fase calcita $\left(\mathrm{CaCO}_{3}\right)$ e o hidróxido de ferro sendo estas confirmadas pelos altos teores de cálcio e ferro apresentado no EFRX (Tabela 3).

Tabela 4 - Numeração das cartas referente às fases presentes na amostra AM1, segundo o banco de dados do JCPDS, e suas respectivas estruturas cristalinas.

\begin{tabular}{c|c|c|c}
\hline Nome do Mineral & Fórmula Química & JCPDS & Estrutura Cristalina \\
\hline Carbonato de calcio & $\mathrm{CaCO}_{3}$ & $70-0095$ & Monoclínico \\
\hline Hidróxido de ferro & $\mathrm{FeOOH}$ & $18-0639$ & Ortorrômbico \\
\hline
\end{tabular}


No difratograma da amostra AM2 (Figura 4) foi detectada uma fase cristalina, a qual apresentou segundo o banco de dados JCPDS (Tabela 5) a fase calcita $\left(\mathrm{CaCO}_{3}\right)$, sendo esta confirmada pelo alto teor de cálcio apresentado no EFRX (Tabela 3).

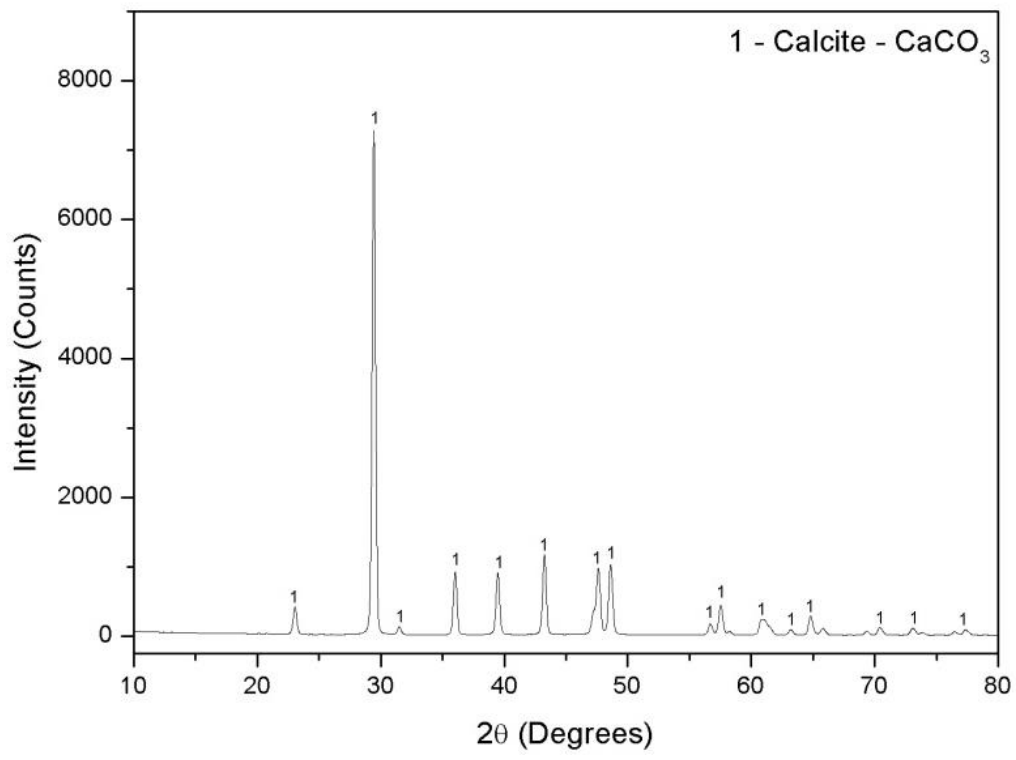

Figura 4 - Difratograma da principal fase presente na amostra AM2.

A formação da calcita presente nas amostras AM1 e AM2, que estavam localizadas na bomba de fundo, pode ter ocorrida devido à presença de íons de $\mathrm{Ca}^{+2}$ e bicarbonatos $\left(\mathrm{HCO}_{3}{ }^{-}\right)$ dissolvidos na água, sendo esta formação favorecida pela diminuição da pressão ou aumento da temperatura no reservatório. Durante a formação da calcita, há a liberação de $\mathrm{CO}_{2}$, que quando associado à água poderá formar o ácido carbônico $\left(\mathrm{H}_{2} \mathrm{CO}_{3}\right)$, ocasionando a corrosão dos componentes metálicos presentes na coluna (SHAMS EL DIN, 2002) como observado na amostra AM1 dada a presença da fase FeOOH.

Tabela 5 - Numeração da carta referente à fase presente na amostra AM2, segundo o banco de dados do JCPDS, e sua respectiva estrutura cristalina.

\begin{tabular}{c|c|c|c}
\hline Nome do Mineral & Fórmula Química & JCPDS & Estrutura Cristalina \\
\hline Calcita & $\mathrm{CaCO}_{3}$ & $72-1652$ & $\begin{array}{c}\text { Rhombohedral rhomb- } \\
\text { centered }\end{array}$ \\
\hline
\end{tabular}

\subsection{MEV}

A análise por microscopia eletrônica de varredura da amostra AM1 utilizando o modo BSE (elétrons retroespalhados) (Figura 5,a) está mostrando, através da tonalidade, que a região " $A$ " e composta por elementos que possuem peso atômico maior quando esta comparada com a região " $B$ ". A região " $A$ " que se encontrava em contato com a bomba de fundo deve ser constituída, em sua maior parte, pelo Hidróxido de ferro $(\mathrm{FeOOH})$ e a " $\mathrm{B}$ " pelo Carbonato de cálcio $\left(\mathrm{CaCO}_{3}\right)$, de acordo com as fases encontradas no difratograma mostrado na Figura 3. 


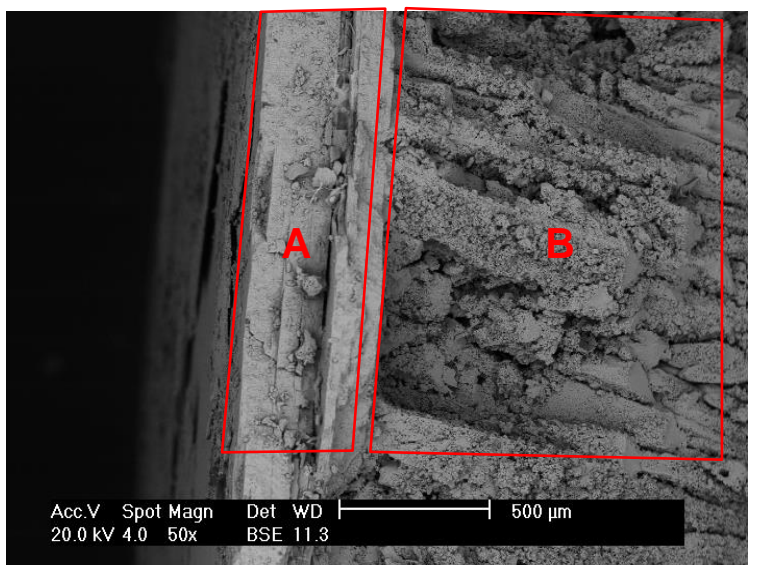

a)

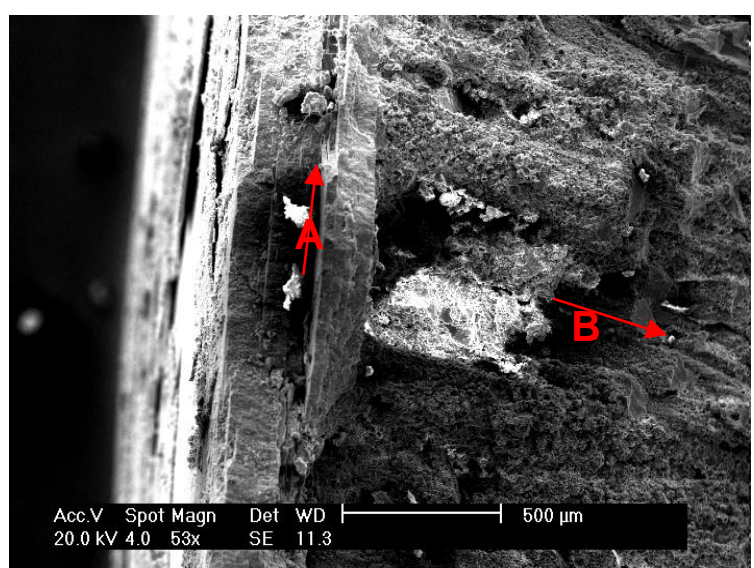

b)

Figura 5 - MEV da amostra AM1 - a) Analise em modo BSE mostrando as regiões com elementos de maior e menor peso atômico, b) Analise em modo SE no qual é observada a morfologia da região.

A análise por meio elétrons secundários (SE) da amostra AM1 (Figura 5,b), é possível visualizar duas formas de crescimento da incrustação: uma longitudinal (seta " $A$ ") e outra perpendicular (seta "B") à superfície da bomba de fundo, a primeira é devido a oxidação da superfície metálica da bomba e a segunda ao crescimento do carbonato de cálcio sendo estas comprovadas pelas analises de EDS (Tabela 6). Também através desta imagem é visto que a região onde se encontra a seta " $A$ " é mais compacta que a região da seta " $B$ ", sendo esta última mais porosa (Per Kjelin, 2003).

Tabela 6-Composição química dos pontos marcados na Figura 5, nos quais foram realizadas as microanálises químicas por EDS.

\begin{tabular}{c|c|c|c|c|c|c|c|c|c|c}
\hline Elemento \% & $\mathrm{C}$ & $\mathrm{O}$ & $\mathrm{Na}$ & $\mathrm{Mg}$ & $\mathrm{Si}$ & $\mathrm{S}$ & $\mathrm{Cl}$ & $\mathrm{K}$ & $\mathrm{Ca}$ & $\mathrm{Fe}$ \\
\hline Região-A & 4,05 & 33,01 & 0,11 & 0,88 & 0,32 & 0 & 0 & 0 & 12,23 & 49,35 \\
\hline Região-B & 22,32 & 16,26 & 0,83 & 1,03 & 1,33 & 0 & 0 & 0 & 32,45 & 25,77 \\
\hline
\end{tabular}

Já a amostra AM2 (Figura 6,a) através da análise de MEV, utilizando-se os elétrons retroespalhados (BSE), é observado que a amostra não possui variação na tonalidade dos elementos presentes, desta forma sua matriz é formada pela predominância de um único elemento, neste caso o cálcio. Já na Figura 6,b tem-se a visualização da imagem com uso dos elétrons secundários (SE), onde observa-se a morfologia dos cristais de calcita.

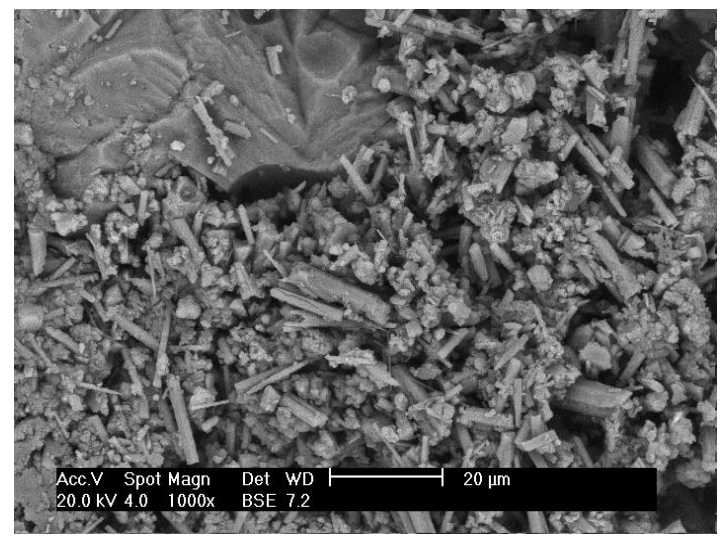

a)

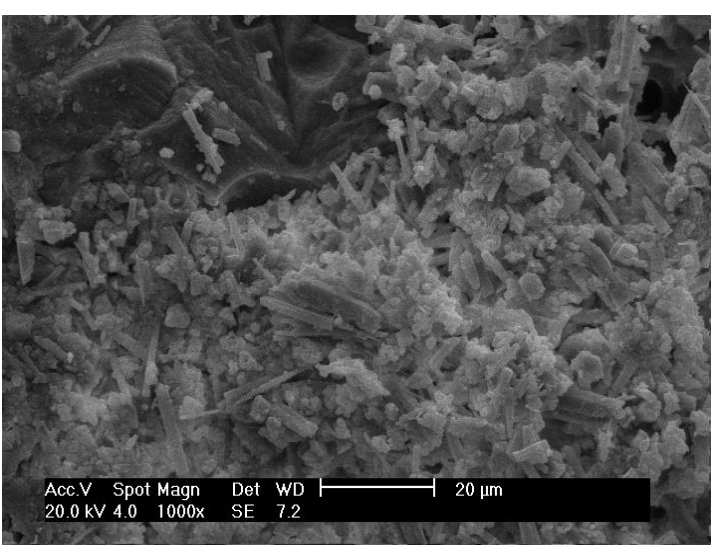

b) 


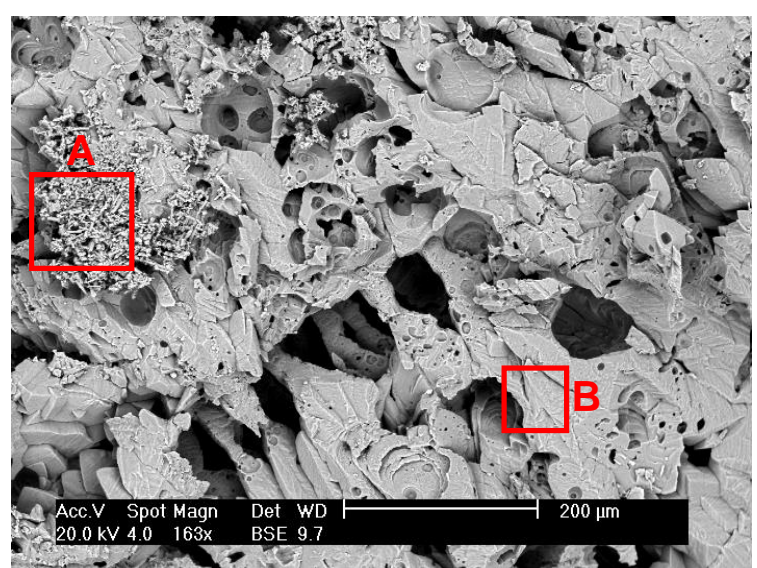

c)

Figura 6- MEV da amostra AM2 - a) BSE mostrando as regiões com elementos de maior e menor peso atômico, b) SE no qual é observada a morfologia de "a" e c) Pontos nos quais foram realizadas as análises químicas por EDS.

Através das Figuras 6, a, b e c, constatou-se a existência de duas principais formas de precipitados: aglomerados e bastões, sendo este último em maior quantidade (indicados pelas setas vermelhas na Figura 6-I). A formação do precipitado deve-se a uma possível mudança no sistema, seja ela temperatura, pressão, solubilidade ou outros fatores que possam afetar a formação do incrustado.

Nas composições químicas, apresentada (Tabela 7) pelos pontos marcados na Figura 6c, é observada uma diminuição na concentração de cálcio do ponto " $A$ " para o " $B$ " e um aumento na concentração do ferro. Desta forma pode-se inferir que os cristais formados são realmente de calcita e o ferro que está incorporado na matriz da amostra AM2 é devido à corrosão das partes metálicas da bomba de fundo. O processo corrosivo é ocasionado entre outros fatores pela liberação do $\mathrm{CO}_{2}$ que ocorre durante a formação da calcita.

Tabela 7- Composição química dos pontos marcados na Figura 6,b, nos quais foram realizadas as microanálises químicas por EDS.

\begin{tabular}{c|c|c|c|c|c|c|c|c|c|c}
\hline Elemento \% & $\mathrm{C}$ & $\mathrm{O}$ & $\mathrm{Na}$ & $\mathrm{Mg}$ & $\mathrm{Si}$ & $\mathrm{S}$ & $\mathrm{Cl}$ & $\mathrm{K}$ & $\mathrm{Ca}$ & $\mathrm{Fe}$ \\
\hline Região A & 7,05 & 18,15 & 1,03 & 1,88 & 0,00 & 0,00 & 0,00 & 0,00 & 65,83 & 6,06 \\
\hline Região B & 21,97 & 16,26 & 0,83 & 1,03 & 1,33 & 0,00 & 0,00 & 0,00 & 36,41 & 22,18 \\
\hline
\end{tabular}

\section{CONCLUSÃO}

Segundo os resultados de extração Soxhlet, as amostras em estudo (AM1 e AM2) apresentaram baixos teores de orgânico influenciados pela pouca ou nenhuma presença de hidrocarbonetos estando estes coerentes em virtude dos poços serem empregados para injeção de água.

Os resultados de DRX e EFRX é possível concluir que ambas as amostras coletadas nas bombas são classificadas como incrustações. A amostra AM2, por ser um produto de incrustação predominantemente carbonático, classificada como incrustação verdadeira (true scale), ou seja, após a formação dos cristais de calcita, estes se depositaram nas superfícies dos equipamentos e componentes das colunas de produção. Já a amostra AM1 pode ser classificada como incrustação 
mista, ou seja, tem-se a deposição de um produto de corrosão (confirmada através da presença de ferro no EFRX da amostra AM1).

\section{REFERÊNCIAS BIBLIOGRÁFICAS}

1. BOIM, J. M. Modelagem Semi-Analítica para Incrustação de Sulfato de Bário em Reservatórios Heterogêneos. Macaé, 2003. Tese de Doutorado-Universidade Estadual do Norte Fluminense - UENF, 2003.

2. CLEGG, J.D, BUCARAM, S.M, HEIN, N.W. Recommendations and Comparisons for Selecting Artificial Lift Methods. JPT - Journal of Petroleum Technology, p.1128-1131, 1163-1167, December 1992.

3. DAHER, J.S. Avaliação de Incrustação de Sais Inorgânicos em Reservatórios Inconsolidados Através da Simulação Numérica. Macaé, 2003. Tese de Doutorado-Universidade Estadual do Norte Fluminense - UENF, 2003.

4. DEMADIS, K. D., MAVREDAKI, E., STATHOULOPOULOU, A., NEOFOTISTOU, E., MANTZARIDIS, C. Industrial water systems: problems, challenges and solutions for the process industries. Desalination, v. 213, p. 38-46, 2007.

5. HEIN, N. W. Beam-Pumping Operations - Problem Solving and Technology Advancements. JPT - Journal of Petroleum Technology, p.330-336, April 1996.

6. JAYASEKERA, A. J., GOODYEAR, S. G. The Development of Heavy Oil Fields in the United Kingdom Continenta Shelf: Past, Present, and Future. SPE Reservoir Evaluation and Engineering, p371-379, October 2000.

7. MARQUES, L. C. C. PEREIRA, A. Z. I. MEIRELLES Jr., R. O. GONÇALVES, M. A. C. How Petrobras Has Faced Oilfield Scale Problems: Evolution of Concepts and Lessons Learned in Campos Basin, Brazil. In: SPE International Symposium on Oilfield Scale held, Aberdeen - UK, 30-31, Janeiro, 2001.

8. MOURA, D. M. A., ARAÚJO, B. A. T., MACHADO, S. F., TÁVORA, M. P., SILVA, A. A. R., CUNHA, J. D., SILVA, D. R. Eficiência de Solventes Orgânicos Apolares na Extração de Produtos de Corrosão. In: VI Corrosion Congress Nace International Latin America Region, 2006, Proceeding LatinCorr 2006, Fortaleza - CE, 1 CD-ROM.

9. PER KJELIN X-ray diffraction and scanning electron microscopy studies of calcium carbonate electrodeposited on a steel surface, Colloids and Surfaces A: Physicochem. Eng. Aspects 212 p. 19-26, (2003).

10. RAMTAD, K., TYDAL, T., ASKVIK, K. M., FOTLAND, P. Predicting Carbonate Scale in Oil Producers From High Temperature Reservoirs. SPE Journal, v. 10, No. 4, p. 363-373, 2005.

11. SHAMS EL DIN, A. M., EL-DAHSHAN, M. E., MOHAMMED, R. A. Inhibition of the Thermal decomposition of $\mathrm{HCO}_{3}^{-} \mathrm{A}$ Novel Approach to the problem of alkaline Scale Formation in Seawater Desalination Plants. Desalination, v. 142, p. 151-159, 2002.

12. TAKÁCKS, G., Sucker-rod Pumping Manual, PennWell Books, 2002.

13. THOMAS, J. E. Fundamentos de Engenharia de Petróleo. Editora Interciência Ltda. 2a ed. Rio de Janeiro/RJ - Brasil, 2004.

14. WOOD, R. J. K. Erosion-corrosion interactions and their effect on marine and offshore materials. Wear, v. 261, p. 1012-1023, 2006. 